\title{
Prader willi syndrome- A case report
}

\author{
Shilpa H. L. ${ }^{1, *}$, Shwetha Odeyar S. ${ }^{2}$, Adarsh E. S. ${ }^{3}$ \\ ${ }^{1,2}$ Assistant Professor, ${ }^{3}$ Associate Professor, Dept. of Anaesthesiology, BGS Global Institute of Medical Sciences, Bengaluru, \\ Karnataka, India
}

*Corresponding Author:

Email: shilpahl@yahoo.com

Received: $14^{\text {th }}$ August, 2017

Accepted: $06^{\text {th }}$ December, 2017

\begin{abstract}
Prader willi syndrome (PWS) is a complex multisystem disorder. PWS mainly affects central nervous system and often involves the hypothalamus. Its major clinical features include neonatal hypotonia, developmental delay, short stature, behavior abnormalities, childhood onset obesity, hypothalamic hypogonadism and characteristic appearance.

Anaesthetic management with general or regional anaesthesia for these patients is difficult mainly due to morbid obesity. The importance of PWS for anesthesiologist and primary physician is estimated by the circumstances as the prevalence of PWS is nearly as frequent as trisomy -21 . We report the anaesthetic management of a 22 year old male patient with genetically proven PWS (small stature, obesity with characteristic behaviour problem) who presented with chronic fissure in ano. Though the clinical course of the patient was uneventful under saddle block. In this case report we discuss our strategy for the prevention of perioperative complications for PWS patients.
\end{abstract}

Keywords: Prader willi syndrome.

\section{Introduction}

Prader willi syndrome (PWS) is a rare genetic disorder with a prevalence of 1/10000-1/30000 resulting from lack of expression of genes on the paternally inherited chromosome $15 \mathrm{q} 11.2-\mathrm{q} 13 .{ }^{1} \mathrm{PWS}$ is a two-staged disorder with a hypotonic early infantile phase and an obese childhood phase. The infantile phase is characterized by failure to thrive, delayed milestones, seizures, fair skin and eyes. The later stage is characterized by obesity due to increased appetite along with behavioural changes, hypotonia, hypogonadal, dental caries, skeletal abnormalities, skin picking and spontaneous bruising. ${ }^{2,3}$

This case report and review of literature on anesthetic management of PWS summarizes the anesthetic implications, importance of early diagnosis, treatment of multiple endocrine and behavioral abnormities which lead to significant improvement in health and developmental outcomes.

\section{Case Report}

A 22-year-old male presented to us with chronic fissure in ano. His history revealed severe skin picking behavior, learning disability, history of repeated styles, poor personal hygiene, psychological and behavioural problems. We observed temper tantrums, impulsivity, stubbornness on the part of the patient. His past medical history revealed seizures at the age of thirteen and patient was on antiepileptics for five years from then. The patient was subjected to cytogenetic analysis and was diagnosed as a case of Prader Willi Syndrome. Family history revealed that the father has similar psychological and behavioural problems.
On examination the patient had characteristic feature of PWS. His height was $150 \mathrm{~cm}$ and weight $80 \mathrm{~kg}$ with a body mass index $35 \mathrm{~kg} / \mathrm{m} 2$ and airway examination revealed Mallampatti Grade. ${ }^{3}$

During the preoperative examination pulse rate was 45beats/min (regular), blood pressure was $106 / 70 \mathrm{~mm}$ $\mathrm{Hg}$. All blood investigations were within normal limits and ECG showed sinus bradycardia. After the history and examination regional anesthesia was planned for the patient.

Preoperatively an 18gauge intravenous (IV) cannula was secured on the right hand of the patient. Inj ranitidine 50mg IV and prophylactic IV dose of $100 \mathrm{mg}$ hydrocortisone was given. Inside the operating room standard monitors -pulse oximeter, noninvasive blood pressure (NIBP) and electrocardiogram were established. Difficult airway cart was kept ready. The patient was made to sit for saddle block and reassured after which under strict asepsis $2.5 \mathrm{cc}$ of $0.5 \%(\mathrm{H})$ bupivacaine was given in L4-L5 interspace using 25gauge quinckes spinal needle after confirming free flow of cerebrospinal fluid. We avoided adjuvants as the patient had a heart rate of 45 beats/minute. Post the spinal procedure the patient was made to sit for 5 minutes and then positioned for surgery. Though the patient was anxious sedation was avoided and the patient was reassured. All precautions to treat inadvertent seizures was kept ready.

The patient with stood the procedure well and post procedure was shifted to the recovery. Hypothermia was avoided both during the procedure and in the postoperative period. Inj diclofenac $75 \mathrm{mg}$ IV and Inj paracetamol $1 \mathrm{gm} \mathrm{IV}$ was given postoperatively. In view of PWS for close observation and therapy patient was 
shifted to a high dependency unit. He had an uneventful course in the hospital and was discharged on third postoperative day.

\section{Discussion}

PWS is caused by a lack of functioning paternally inherited genes on the long arm of chromosome 15 (deletion of chromosome 15q 11-q). The incidence of PWS is about 1 in $15,000-25,000$ births, the male to female ratio is $3: 2{ }^{4}$

The syndrome progresses during childhood and is characterized by hyperphagia leading to obesity plus endocrine abnormalities including hypogonadism and diabetes mellitus .Other associated endocrine abnormality contribute to the clinical picture of short stature secondary to growth hormone deficiency and a decreased bone mineral density is noted 1 . Patients with PWS with a deletion of genotype showed a trend toward developing seizures and were easy to control with single antiepileptic drug. ${ }^{5}$

Children with PWS frequently suffer from restrictive lung disease because of hypotonia, obesity and kyphoscoliosis. Equipment's should be available to deal with the difficult to intubate/ventilate scenario, even when regional anesthesia is planned. Anesthetic concerns in these patients of PWS mainly centers on obesity and related complications like diabetes mellitus, hypertension and sleep apnea. ${ }^{6}$ Due to food seeking behavior, NPO status cannot be assured and these patients to be treated as full stomach and higher incidence of hiatus hernia secondary to obesity all contribute to increased risk of gastric aspiration, recurrent respiratory infections and dental caries. 10$17 \%$ incidence of rumination risk of aspiration of gastric contents in PWS. ${ }^{7}$

Patients with PWS present with decreased gastric motility heightening the risk of gastric aspiration, hence inj ranitidine and inj metachlopromide one hour prior to anaesthesia should be considered as an effort to reduce gastric secretion and to increase intestinal motility. It has been noted that Central adrenal insufficiency affects as many as $60 \%$ of PWS patients.

Supplementation of stress dose of hydrocortisone is required to prevent post-operative laryngospasm and bronchospasm. ${ }^{8}$ Both general and regional anaesthesia are challenging. The use of general anaesthesia may predispose to difficult airway management and landmarks for regional anesthesia may be obscured due to morbid obesity as was with our present case. Though regional anaesthesia -saddle block was performed in this case, ready access to deal difficult airway was kept ready. Regional anesthetic techniques offer intraoperative anaesthesia care and postoperative analgesia and provides means of limiting the need for opioids .It has been suggested that regional techniques and non steroidal anti inflammatory drug are beneficial in the immediate post-operative period.

\section{Conclusion}

In summary, preoperative evaluation of PWS patients should be individualized and special attention must be paid to those patients complicated with severe OSA. Anticipation and preparedness for intraoperative challenges mainly airway management, hypotonia, hypothermia and difficulty in regional techniques. Postoperative intensive care is recommended for PWS with severe OSA to prevent sleep - related respiratory complication. Overuse of sedation in the postoperative period to be avoided and prolonged supervision of the respiratory function, blood glucose and body temperature is recommended.

To conclude early diagnosis, referral to psychologist and comprehensive care of patients with PWS have better outcome.

\section{References}

1. Emerick JE, Vogt KS. Endocrine manifestations and management of Prader-Willi syndrome. Int J Pediatr Endocrinol 2013;21:14.

2. Dearlove OR, Dobson A, Super M. Anaesthesia and Prader-Willi syndrome. Paediatr Anaesth 1998;8(3):26771.

3. Yatish Bevinaguddaiah, Shivakumar shivanna, Tejesh c anandaswamy, Vinayak pujari. Prader willi syndrome with oculocutaneous albinism: anaesthetic implications and management. National Journal of laboratory Medicine 2014;3(2):13-15

4. Peter Biro. Anaesthesia in a patient with Prader-Willi syndrome and severe burn injury: a case report. J Rom Anest Terap Int 2011;18:149-152

5. Vendrame M1, Maski KP, Chatterjee M, Heshmati A, Krishnamoorthy K, Tan WH, Kothare SV. Epilepsy in Prader-Willi syndrome: clinical characteristics and correlation to genotype. Epilepsy Behav 2010;19(3):30610.

6. J W Mackenzie. Anaesthesia and the Prader-Willi syndrome. J R Soc Med. 1991 Apr; 84(4):239.

7. Tod B. Sloan, Celia I. Kaye. Rumination Risk of Aspiration of Gastric Contents in the PraderWilli Syndrome. Anesth Analg 1991;73:492-95.

8. De Lind van Wijngaarden RF, Otten BJ, Festen DA, Joosten KF, de Jong FH, Sweep FC et al. High prevalence of central adrenal insufficiency in patients with prader willi syndrome. J Clin Endocrinol Metab 2008;93(5):1649-54. 